\title{
Conserver ensemble patrimoines naturel et culturel
}

L'exemplarité des ruines féodales des Vosges du Nord

Lucie Dupré

\section{(2) OpenEdition \\ Journals}

Édition électronique

URL : https://journals.openedition.org/tc/3943

DOI : $10.4000 /$ tc. 3943

ISSN : 1952-420X

\section{Éditeur}

Éditions de l'EHESS

Édition imprimée

Date de publication : 31 décembre 2008

Pagination : 84-101

ISSN : 0248-6016

\section{Référence électronique}

Lucie Dupré, «Conserver ensemble patrimoines naturel et culturel », Techniques \& Culture [En ligne],

50 | 2008, mis en ligne le 31 décembre 2010, consulté le 29 septembre 2022. URL : http://

journals.openedition.org/tc/3943; DOI : https://doi.org/10.4000/tc.3943 


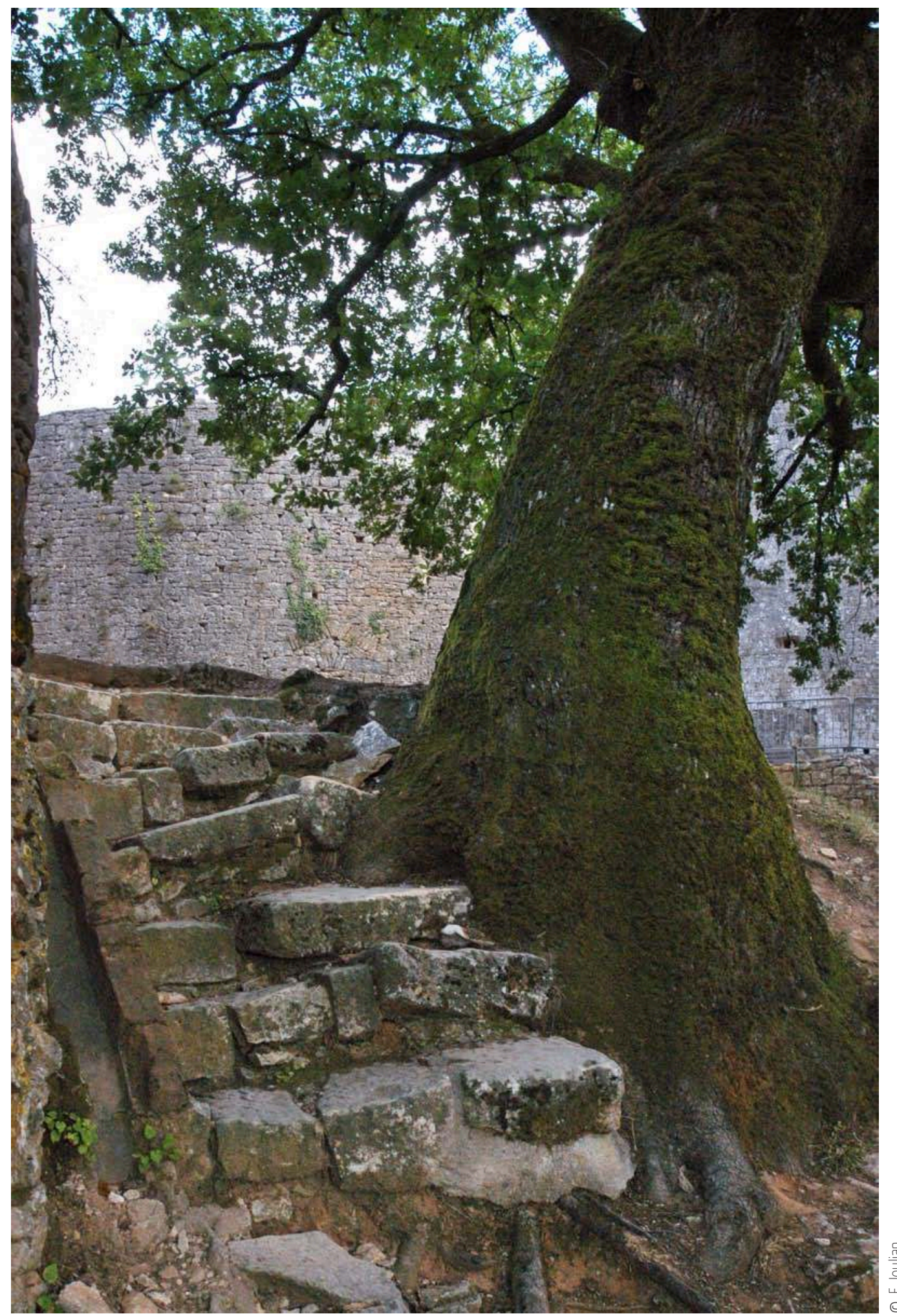




\title{
CONSERVER ENSEMBLE PATRIMOINES NATUREL ET CULTUREL
}

\section{L'exemplarité des ruines féodales des Vosges du Nord}

\author{
Les ruines féodales des Vosges du Nord constituent un patrimoine exemplaire \\ de l'intrication forte, complexe et parfois problématique entre la nature et la \\ culture. Comment protéger dans le même temps la nature et la culture dans de \\ tels sites précisément fondés sur l'imbrication de ces deux dimensions?
}

Selon qu'il est naturel ou culturel, le patrimoine a ses institutions propres, son administration, ses modes de traitement et de conservation spécifiques, son régime juridique, ses experts, son public... Il en est de même selon qu'il est qualifié d'« ordinaire » ou de « remarquable ». Certains objets patrimoniaux démentent toutefois une partition aussi tranchée: ils procèdent d'une forte imbrication nature-culture qui fonde précisément leur qualité et leur singularité patrimoniales. Les nombreuses ruines féodales ${ }^{1}$ qui ponctuent le territoire du Parc naturel régional des Vosges du Nord ${ }^{2}$ sont à ce titre des témoignages exemplaires de la nécessité d'établir une approche conjointe de leur grandeur patrimoniale.

Ces ruines appartiennent au ministère de l'Agriculture, via les services locaux de l'Office national des forêts (ONF) qui en assurent la gestion en domaine sylvicole public. En domaine sylvicole privé, elles sont gérées par le Groupement de Gestion Sylvicole des Vosges du Nord dont le siège social est basé à Dambach (Alsace). Dans le même temps, et à l'exception de l'un d'entre eux, ces vestiges partagent une caractéristique commune: ils sont tous protégés au titre des Monuments historiques par classement 


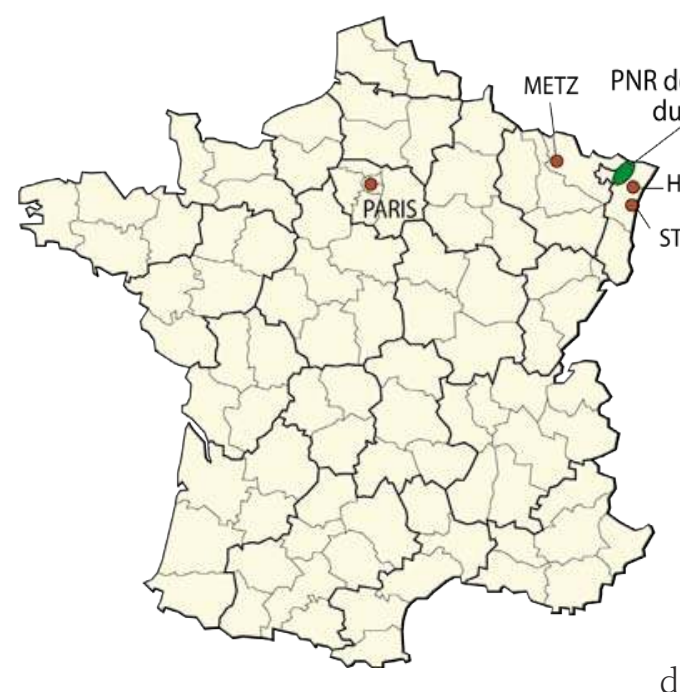

ou inscription à l'inventaire supplémentaire. Les ruines qui nous intéressent - que j'ai appelées « châteaux de forêt »-présentent en outre la particularité d'être souvent situées au sommet de collines boisées, au cour de territoires forestiers (Fig. 1). On le devine, cette situation s'accompagne d'une tension forte dans la conservation de ces sites. Les moyens à mettre en œuvre et les enjeux associés à la gestion et à

l'ouverture de ces ruines de château pour un public très diversifié font apparaître toute leur complexité patrimoniale. Car l'intérêt de ces ruines est de se situer à la convergence d'un faisceau d'intérêts naturels et culturels déterminés principalement par leurs différents usagers. Une approche de leur complexité suppose donc de s'intéresser aux différents usages de ces bâtiments, du moins ceux qui font l'objet de revendications.

On trouve dans la conceptualisation pionnière proposée au début du siècle par Aloïs Riegl une ressource intéressante pour s'emparer de cette question (Riegl 1984 [1903]). Selon lui, la ruine est animée de deux valeurs - la valeur d'ancienneté et la valeur historique $e^{3}$ - amenées à se compléter ou à entrer en tension lors de la « réception monumentale », mais aussi lors des choix des différents traitements réservés à l'objet patrimonial - et qui visent à instruire la prise en charge de son devenir. La valeur d'ancienneté et la valeur historique procèdent d'un objet dont le caractère hybride conduit à reconsidérer le partage classiquement opéré dans l'appréhension des objets patrimoniaux, à savoir leurs caractères naturel ou culturel, ici précisément conjoints. En effet, en introduisant la notion de valeur d'ancienneté, Aloïs Riegl lie intimement deux champs que les sciences ont pris soin de dissocier et d'opposer dans le traitement de leurs objets respectifs: la nature et la culture. Il invite donc de façon pressante à reformuler le dialogue souvent évité entre les dynamiques naturelles et culturelles à la croisée desquelles cette valeur se déploie. La gestion de ces hauts lieux de la

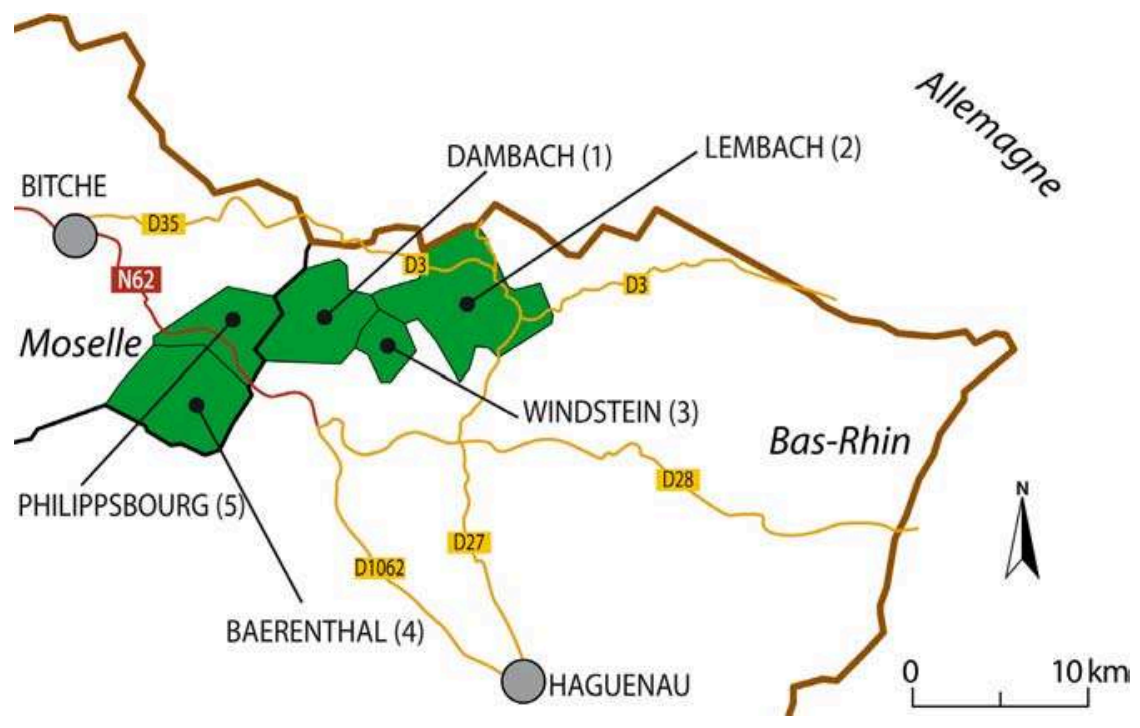

Ruines de châteaux:

(1) Hohenfels

(2) Fleckenstein

(3) Nouveau et Vieux Windstein

(4) Ramstein et Grand-Arnsourg

(5) Falkenstein

Cartographie Catherine Lefebvre (INRA - SAE2 - MONA)

(Fig. 1) 
culture locale confronte les acteurs qui y sont associés, qu'il s'agisse des propriétaires, des gestionnaires ou des collectifs engagés dans leur protection et leur valorisation, à la prise en compte de ce caractère hybride; lequel invite à déployer des dispositifs patrimoniaux excédant largement la seule question architecturale, comme on va le voir.

Après avoir exposé rapidement l'histoire et les particularités du tourisme castral - ces dernières orientant de façon décisive les modes de gestion de ces sites - on examinera de manière plus approfondie les tensions qui se font jour dès lors que surgissent sur la scène patrimoniale des acteurs non humains mais pourtant bien vivants et souvent représentés par des porte-parole qui défendent leurs droits. Ainsi, il sera d'abord question du problématique traitement des végétaux et de leur alliance avec le bâtiment qui scelle la singularité des ruines. Puis, j'évoquerai une autre catégorie d'acteurs non humains: les faucons pèlerin. Dans l'imaginaire, ces oiseaux sont peut-être moins fortement associés aux ruines de châteaux que la végétation romantique qui les caractérise, mais leur présence dans certains sites des Vosges du Nord met face à face des collectifs que rien ne rassemble par ailleurs et qui vont devoir trouver un accord dans la pratique des lieux.

\section{Des objets démocratiques: naissance, développement et singularité d'un tourisme castral}

Depuis le premier quart du XIX ${ }^{e}$ siècle, artistes, intellectuels et acteurs touristiques n'ont pas manqué de se faire l'écho et de vanter le charme romantique des ruines des Vosges du Nord, relayés en cela par deux institutions qui ont contribué à les ériger au titre des curiosités locales.

\section{Rendre accessibles les forêts et leurs ruines}

Créé en 1872, le Club Vosgien s'est donné pour tâche d'ouvrir le massif forestier et d'y aménager un certain nombre de sentiers conduisant dans bien des cas à des ruines de châteaux, sur lesquelles des générations de bénévoles n'ont cessé d'intervenir. La plus importante association à vocation touristique de France a ainsi largement contribué à socialiser la forêt

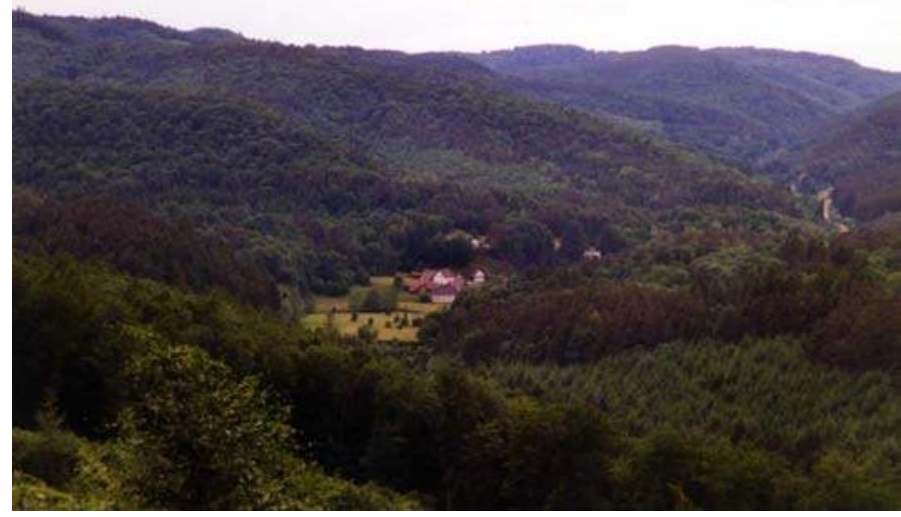

Une vue imprenable

Les châteaux offrent une vue sur le vaste territoire forestier des Vosges du Nord. Ici, la vallée de la Zinsel du Nord, depuis le château du Grand Arnsbourg (Baerenthal).

(Fig. 2) en la rendant accessible à une forme de tourisme, alors réservé à une élite téméraire et savante issue des grandes villes voisines, en quête de « point de vue » sur un territoire plus vaste (Huck 1972 : 233) (Fig. 2). Les efforts du Club Vosgien n'ont pas été isolés. Les professionnels du tourisme thermal, dont la ville de Niederbronn-les-Bains a fait sa spécialité depuis le milieu du XIX ${ }^{e}$ siècle, ont à leur manière contribué à faire découvrir les ruines 

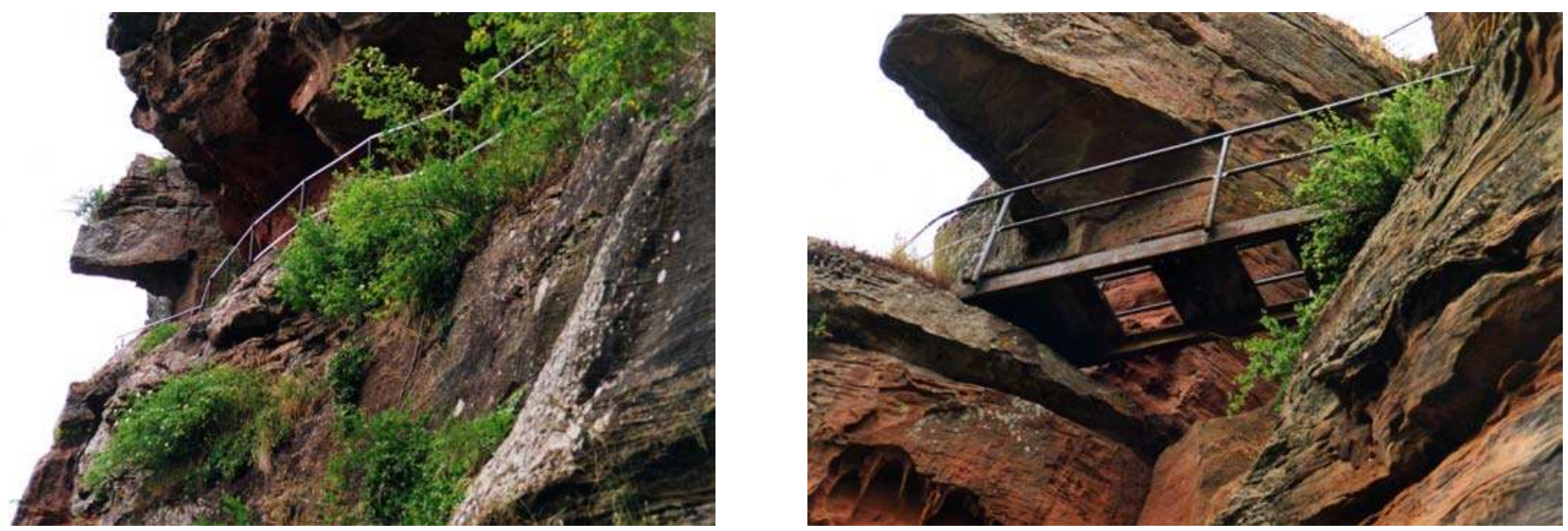

\section{Rendre les ruines accessibles}

On doit au Club vosgien les premières infrastructures permettant de visiter les ruines de châteaux : panneaux informatifs, tables d'orientation installées au sommet de certaines tours, escaliers et passerelles, rambardes de sécurité, aménagement d’aires de piquenique à proximité.... Ces équipements, généralement faits avec les moyens du bord, sont souvent vétustes et ne peuvent être régulièrement entretenus faute de moyens. À ce titre, certains sites estimés dangereux ont été fermés par leurs gestionnaires, suscitant localement une très vive émotion tant l'attachement à ces ruines est fort.

(Fig. 3 a et b)

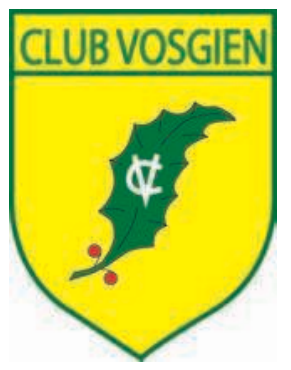

\section{Emblème du Club Vosgien}

Le Club vosgien est un acteur majeur du développement d'une forme de tourisme populaire dans tout l'Est de la France. II regroupe aujourd'hui encore de très nombreux adhérents dans chaque village et organise régulièrement excursions, voyages et visites culturelles.

(Fig. 4) en offrant une activité touristique à une population de curistes fortunés et curieux. Depuis l'après-guerre, la démocratisation des loisirs, l'évolution des transports et l'amélioration des voies de communication, conjuguées aux efforts presque ininterrompus du Club Vosgien, ont contribué à rendre de plus en plus attractives et accessibles les forêts vosgiennes et leurs ruines (Fig. 4). Une forme de tourisme populaire s'est largement développée et qui occupe aujourd'hui le cœur des politiques locales (Fig. 3a et 3b). D'aucuns y voient la marque d'une revanche de l'histoire: Georges Bischoff estime que les enjeux liés à l'émergence de ce tourisme populaire renvoient sans ambiguité à la réappropriation collective de lieux associés à la puissante figure de seigneurs jouissant de privilèges. Ce renversement de situation - le château n'est pas réservé à un cercle réduit d'individus mais doit être ouvert au plus grand nombre - le conduit à conclure que la ruine est « le symbole des libertés populaires. À commencer d'ailleurs par la liberté de circulation dans ces sites reconquis $»$ (Bischoff 1989: 63).

De fait, ces ruines s'adressent aujourd'hui à un public particulièrement hétérogène en terme de classe d'âge ou de profil socioculturel. En effet, dans la plupart des cas ${ }^{4}$, aucun droit d'entrée n'est exigé et l'effort consenti pour y accéder n'est pas discriminatoire: la visite relève d'une pratique ordinaire de la nature. Enfin et surtout, leur caractère hybride reposant sur l'association entre la valeur d'ancienneté et la valeur historique n'exclut a priori aucun type de public. La réception sensible du monument se combine en effet sans tension avec une forme de réception qui peut, dans certains cas, être plus érudite (Dupré 2001).

\section{La réception sensible de la ruine: un tourisme ordinaire}

Pour Aloïs Riegl, le monument ancien est davantage apte à dégager une « ambiance » que le monument moderne. Lambiance est un terme qui réfère à la qualité immatérielle de l'ajustement entre des hommes et des objets matériels, à l'intimité de sa perception dans l'instant de la relation. Les châteaux de forêt sont dits sauvages, isolés, mystérieux; ils sont présentés comme des lieux naturels bénéficiant d'une situation qui les distingue des châteaux de village, dans la mesure où ils coexistent avec une solitude boisée propice à les rendre encore plus mystérieux, plus 
étrangers et donc plus naturels. Objets culturels rendus à la nature, ils sont tout entiers portés par leur écrin de verdure qui nous les cache: ils ne s'offrent pas sans investissement, sans que l'on ne consente à quelques efforts, dès lors largement récompensés - l'ambiance est à ce prix. La ruine est un bâtiment à ciel ouvert: le vent, la brume, la pluie, les bruits, bref le monde de la forêt, élargissent la palette sensorielle mobilisée dans la réception monumentale. La dimension contemplative de cette expérience est d'autant plus forte que d'autres éléments, comme on va le voir, participent de la qualité immatérielle de ces lieux plongés dans une sorte de paix propice à des rêveries solitaires.

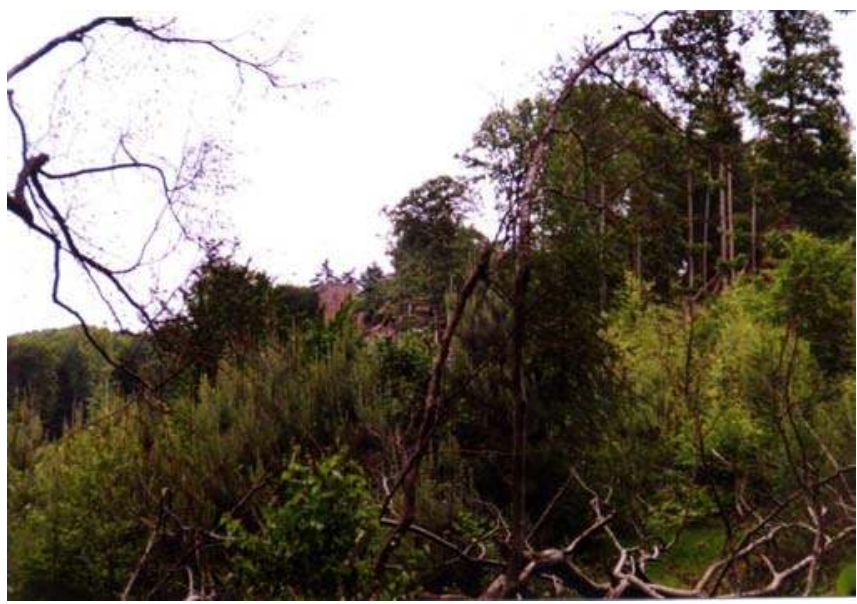

Lanalyse des réponses faites à un questionnaire diffusé sur deux communes lorraines (Baerenthal et Philippsbourg) comptant des ruines emblématiques montre que les visiteurs associent très clairement la ruine à la détente, à la contemplation, à la promenade silencieuse: on « regarde, rêve, admire ». Ces informations convergent tout à fait avec la manière de pratiquer la forêt. L'une et l'autre pratiques, celle de la forêt et celle du château, se combinent étroitement parce que l'une et l'autre font corps. La ruine, a écrit le sociologue allemand Georg Simmel ${ }^{5}$, « s'incorpore au paysage environnant, s'y greffe comme l'arbre ou la pierre. [...] Les influences de la pluie et du soleil, de la chaleur et du froid ainsi que de l'envahissement par la végétation ont amené l'édifice qui leur était abandonné à une conformité de tons avec le paysage soumis aux mêmes destinées: elles ont nivelé les contours, s'élevant autrefois en opposition avec la ligne du sol, dans une paisible et harmonieuse unité » (Simmel 1912: 121). Nuançons cette assertion.

Des ruines en leurs forêts

La végétation fait-elle écran ou sert-elle décrin aux ruines? II s'agit d'un débat ancien qui partage encore aujourd'hui gestionnaires et visiteurs. En été comme au printemps, les ruines sont difficiles à apercevoir et il faut parfois les chercher longtemps du regard pour finalement les distinguer dans le paysage. Ici les tours du Falkenstein (commune de Phillipsbourg) ci-dessous et celle du Grand-Arnsbourg (commune de Baerenthal) ci-dessus qui émergent à peine de la végétation.

(Fig. 5 et 6) Si la ruine se fond dans le paysage, elle ne s'y perd pas pour autant bien que de nombreux édifices soient souvent envahis par une végétation dense (Fig. 5 et 6). En effet, les éléments naturels qui caractérisent les châteaux de forêt marquent une certaine rupture avec ceux des forêts alentour, autant par leur forme que par leur nature. Ainsi, les arbres perchés au faîte des plateformes sommitales sont presque nanifiés: le manque d'éléments nutritifs les a contraints à une croissance très lente puisqu'ils sont obligés de lancer leurs racines noueuses, telles des bras de pieuvre géante, dans les interstices de la pierre. De plus, les ruines hébergent souvent une flore particulière dont la présence s'explique par des facteurs anthropiques autant contemporains - les visiteurs apportent à leur insu des semences exogènes -, qu'historiques puisque certaines espèces médicinales ou alimentaires observables aujourd'hui pourraient être issues des anciens jardins médiévaux ${ }^{6}$.

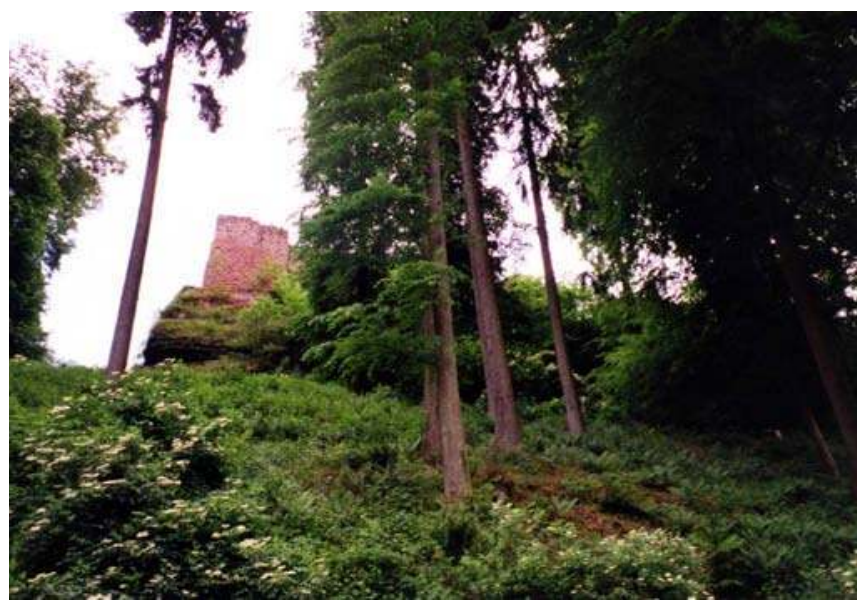



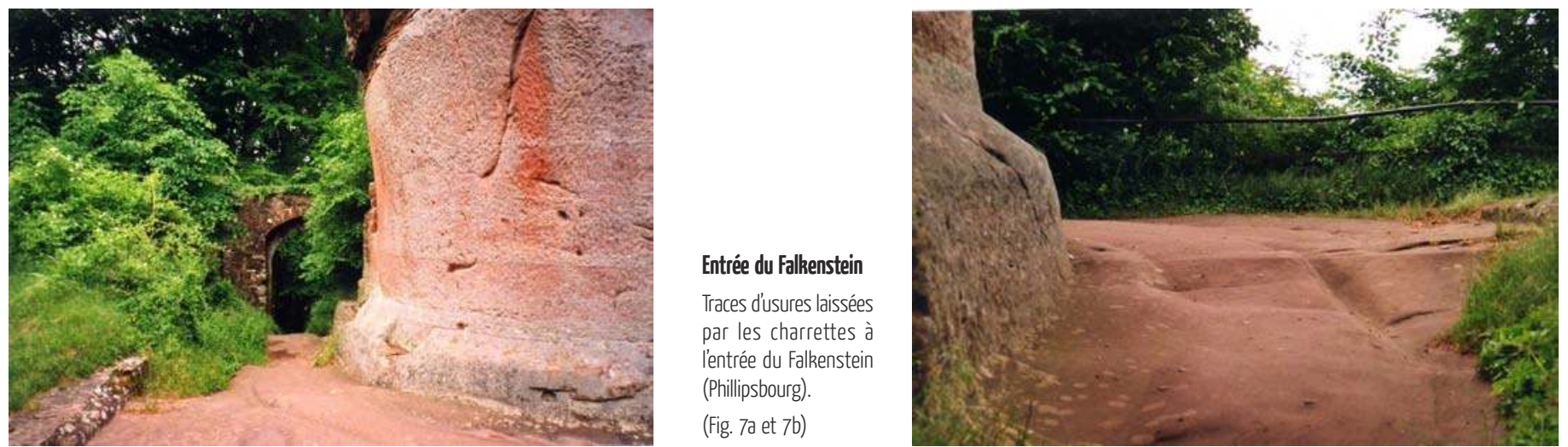

Entrée du Falkenstein

Traces d'usures laissées par les charrettes à l'entrée du Falkenstein (Phillipsbourg).

(Fig. 7a et 7b)
De la ruine pacifiée par le temps au long cours se dégage une certaine douceur, presque sécurisante, tout au moins rassurante. La roche et le rocher usés par le temps renvoient à une érosion, d'ailleurs autant naturelle qu'anthropique ${ }^{7}$, porteuse d'émotion (Fig. 7a et 7b). L'édifice construit par l'homme qui l'a dressé vers le haut, est ramené vers le bas par l'action mécanique de la nature. Mais, précise Georg Simmel « tant que l'on peut parler de ruines et non de monceaux de pierre, la nature ne permet pas que l'œuvre tombe à l'état amorphe de la matière brute [puisque] une nouvelle forme est née, qui, du point de vue de la nature, est absolument sensée, [...] différenciée » (Simmel 1912 : 120). Cet équilibre, fragilisé et menacé par le temps qui passe avec son cortège d'agents climatiques, n'inspire d'une façon générale, ni pitié ni effroi ${ }^{8}$. Les sentiments de paix et d'harmonie désamorcent tout ce qui pourrait s'apparenter à l'idée d'un certain danger dans ces lieux que l'on dit par ailleurs « sauvages » et « isolés ». La « sauvagerie » étant entendue au sens étymologique évoque le lien avec la forêt (silva) socialisée ici par la ruine. On ne s'étonnera donc pas que les châteaux de forêt se fréquentent comme la forêt: en silence.

Cette réception sensible et ordinaire, qui passe également par un corpus de légendes venant tisser et renforcer la dimension magique des ruines, n'entre pas en tension avec le savoir historien vers lequel certaines manières de recevoir le monument ancien offrent même une transition. En revanche, en ce qui concerne la gestion des lieux, la valeur historique des ruines semble gouverner les choix qui président à leur prise en charge. Le second temps de ce texte se propose donc d'interroger la prise en charge, nécessairement complexe de ces sites car leur intérêt est précisément d'allier la nature à la culture (Fig. 8).

\section{La prise en compte des acteurs non humains dans le traitement patrimonial des ruines}

La ruine ne saurait être réduite au symbole du temps qui s'écoule: elle est un objet concret fait de pierre, de lierre et de fer, soumis à une forte fréquentation. La singularité de la ruine, en tant qu'objet hybride, se déploie non sans poser quelques problèmes dès lors que les gestionnaires se proposent de définir des politiques de conservation. 


\section{Composer avec les lois de la ruine}

La ruine est irriguée par ces deux valeurs, la valeur d'ancienneté et la valeur historique, dont Aloïs Riegl a montré le complexe entrecroisement (Riegl 1984). Chacune d'elles réfère à une loi; la première renvoie à celle de la dégradation et du « mouvement cyclique » puisque ce sont précisément les traces d'usure, de patine et d'érosion qui confèrent au bâtiment sa valeur en tant que monument ancien. La seconde relève de la loi de la création et entend porter témoignage de la volonté artistique, technique et culturelle qui a présidé à la naissance du monument, voire à ses successives transformations, en luttant contre le mouvement inéluctable de dégradation. Le monument est ainsi à la fois menacé et mis en valeur par cet environnement naturel. D'un côté, le culte de la valeur d'ancienneté commande de ne pas intervenir et d'accepter le destin naturel de la ruine et s'élève tout autant contre sa « destruction violente par la main de l'homme, intervention sacrilège sur le processus de décomposition naturelle» (Ibid. : 69). De l'autre, le culte de la valeur historique demande de « veiller à conserver les monuments en leur état présent » (Ibid.: 75), de telle manière que soit préservée leur qualité de « document authentique ». Ainsi, la question de la conservation est le lieu de l'opposition entre les intérêts de chaque valeur (Ibid.): pour les deux, le monument est intouchable, mais pas de la même manière. À charge donc pour les générations qui « reçoivent » le monument de déterminer sa valeur prégnante et le « culte » dont il doit faire l'objet, voire, en l'occurrence, de tenter un compromis qui concilie ces valeurs dans le présent des collectifs, au nom du caractère hybride de la ruine, objet à la fois «naturel » et « culturel » (Fig. 9 et 10).

\section{Sanctuariser la ruine ou conserver la culture: pour une ruine expérimentale}

La comparaison avec la gestion des espaces naturels mérite d'être explorée. En effet, la «protection » et la « préservation » d'espaces naturels s'accompagnent souvent de la mise en place de mesures réglementaires visant à atténuer les conséquences de l'action humaine sur la biodiversité, soit dans le sens de son appauvrissement, soit au niveau des conditions mêmes de sa reproduction. Or, dans le cas des ruines, c'est précisément la non-intervention humaine qui serait nuisible au bâtiment puisque son évolution naturelle conduit à terme à sa désagrégation totale, marquant une expulsion - vécue comme une défaite - du champ culturel. D'un côté, c'est l'action de l'homme qui est combattue, de l'autre, c'est l'action de la nature. Pour autant, si l'on poursuit cette comparaison, on constate que la saisie patrimoniale de ces objets se fait selon un même principe de « mise à l'écart»; seuls les termes changent: muséification pour les uns, sanctuarisation pour les autres. La symétrie évidente entre ces d'objets appartenant tous deux au domaine patrimonial invite à faire l'hypothèse que certaines ruines de château

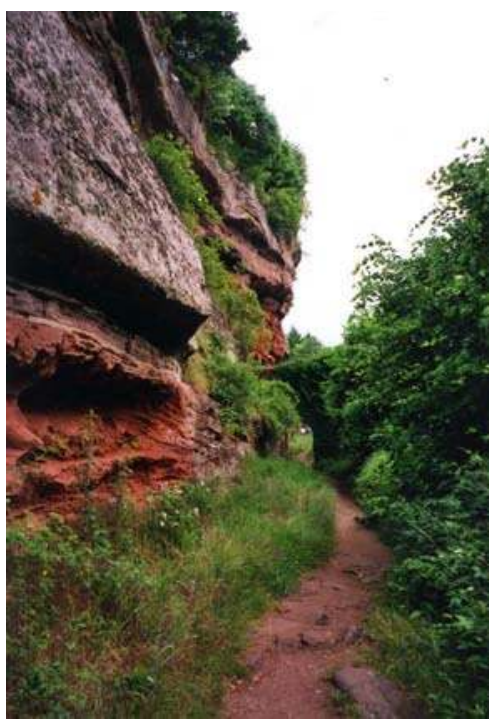

Longer la ruine

L'entretien des accès est variable selon les sites et leur fréquentation. Le principal problème est la maitrise de la végétation qui referme les chemins mais qui confère dans le même temps à la ruine un charme particulier. Ci-dessus, le chemin lon-

geant le Falkenstein, un château phare de la région, est régulièrement entretenu. Ci-dessous, le chemin longeant le piton gréseux sur lequel le Grand Arnsbourg a été édifié est en passe de se

refermer.

(Fig. 9 et 10)

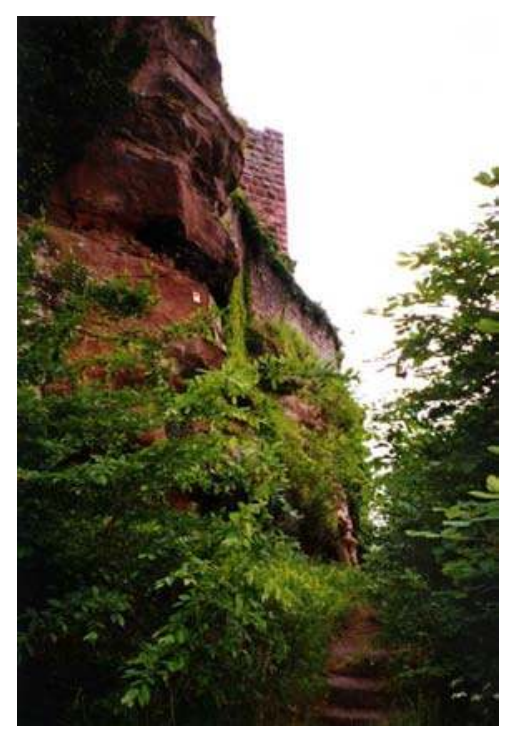




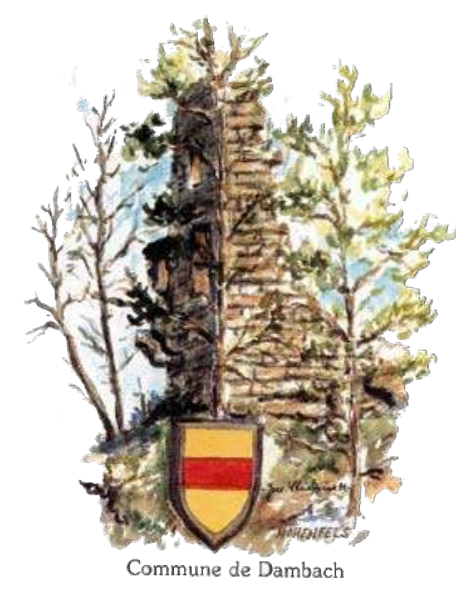

Carte de visite de la commune de Dambach (Alsace)

Le Hohenfells est devenu l'emblème de Dambach. Bien quil ne reste quiun piton de grès, ce château redevenu rocher est très fréquenté et les habitants y sont très attachés.

(Fig. 11) pourraient être, de façon expérimentale, livrées à leur destin de ruine, quitte, pour cela, à en faire des objets interdits d'usage, offerts à la seule contemplation de leur décrépitude.

Ce scénario se heurte à plusieurs objections. Les experts consultés peuvent difficilement abonder dans ce sens puisque leur mission professionnelle les charge précisément de veiller à l'entretien et à l'intégrité d'un patrimoine reconnu comme historique. Ainsi, du côté des Monuments historiques (direction régionale des Affaires culturelles d'Alsace), si on envisage cette perspective, c'est en terme d'échec: «Ça se passera sûrement mais ça ne sera pas un choix. Ça sera par défaut. Il y a des ruines qui deviendront des vraies ruines par absence de choix». La ruine « acceptable » n'est donc pas une « vraie ruine»: elle est un "château en ruine » qui combine encore valeur d'ancienneté et valeur historique. La "vraie ruine », quant à elle, serait un objet entièrement reconquis par la nature: un tas de cailloux masqué par la végétation, pointant l'échec des experts et des professionnels confrontés à l'ampleur de la tâche, à la lourdeur financière des opérations, etc. Sa valeur historique aurait totalement disparu, mais dans le même temps elle perdrait sa valeur d'ancienneté. En effet, comme le souligne Aloïs Riegl, soumise à la loi de la dégradation, la valeur d'ancienneté « travaille à sa propre perte » mais elle exige pour s'exercer, un support minimal (Riegl 1984 : 70). En l'occurrence, la vraie ruine se confondrait dans la plupart des cas au piton gréseux sur lequel les châteaux ont été édifiés (Fig. 11).

Si dans les Vosges du Nord, les pitons gréseux ne manquent pas et sont susceptibles d'émarger à la catégorie de « patrimoine naturel remarquable », les châteaux de forêt en sont bien distingués: bien que souvent décrits comme naturels et sauvages, ils sont clairement associés, dans les discours, à un patrimoine culturel (Fig. 12). Dans la mesure où la «vraie ruine » a perdu toute valeur de remémoration, laisser les châteaux aboutir à cet état serait alors aller à contresens de leur qualification. En outre, la perspective de la « vraie ruine » implique que le site soit libéré de toute préoccupation scientifique - et en cela, le caractère expérimental de la ruine se distingue nettement du caractère expérimental de la Réserve biologique intégrale $e^{9}$ en tant que ce dispositif vise précisément à créer un lieu d'expériences scientifiques. Refuser que la ruine vive son destin naturel et la soustraire à ce qui est dès lors vécu comme une déchéance culturelle contraint l'homme à intervenir sur la scène patrimoniale. Ici, se déploie ce que Marc Guillaume définit comme un « jeu avec la temporalité pour maintenir en équilibre les forces de destruction naturelles et l'acte de création initial» (Guillaume 1980 : 118). Il s'agit alors de pratiquer des soins «palliatifs » et conservatoires $^{10}$ qui ne font qu'accorder un sursis à la ruine puisqu'ils repoussent le problème à la génération 
suivante, laquelle sera libre de se saisir à sa manière du patrimoine transmis - l'intervention n'étant jamais définitive. La ruine doit donc « rester une ruine».

\section{Maintenir la ruine en état}

Mis à part quelques véritables projets de restauration (le Lichtenberg, le Fleckenstein, etc.), la grande majorité des éléments du corpus vosgien est éligible à un traitement ponctuel de sauvegarde des éléments architecturaux (un cadre de fenêtre, une poivrière, la pierre qui marque le seuil d'une porte...) signifiant la spécificité du bâtiment (Fig. 13, 14 et 15). Ce mode d'intervention permet de conserver en l'état le monument de telle sorte que, pour reprendre les mots de John Ruskin, «plus d'une génération naîtra encore et disparaîtra sous son ombre ( Ruskin 1929 : 161). Il faut pourtant remarquer que, bien que minimaliste, ce traitement est loin d'être appliqué à l'ensemble des ruines, faute de crédits et souvent de volonté politique. D'un point de vue souvent exprimé par les acteurs ordinaires - et qui va à l'encontre d'une conception romantique de la ruine - la végétation apparaît davantage comme un écran masquant ce qui contribue à la grandeur du monument, que comme un écrin venant en magnifier le caractère hybride. Au plan de l'expertise technique du monument historique, la gestion de la végétation apparaît bien plus complexe et nécessite chaque fois des choix circonstanciés. La végétation et les fondations sont ainsi parfois si étroitement associées qu'il est difficile ou dangereux de les dissocier: on ne sait plus qui tient l'autre, on n'y touche donc pas. Le monde végétal change ici de statut: d'agent destructeur les racines se glissent dans chaque interstice à la recherche de quelques éléments nutritifs, soulevant au passage les pierres et marquant le début d'un cycle de délabrement qui ne pourra être que temporairement stoppé -, il devient un allié dans la conservation de l'objet architectural. En même temps qu'il se développe sur la ruine et menace de la dégrader, il l'enveloppe et la maintient. En d'autres termes, il la redéfinit à la fois symboliquement et techniquement. Si les végétaux sont ordinairement associés à l'objet " ruine », une autre catégorie d'acteurs vivants non humains, moins prévisible, oblige cependant à inventer des politiques de gestion originale de certains de ces sites. En effet, quelques ruines des Vosges du Nord mettent face à face d'une part, le faucon pèlerin et ses porte-parole et d'autre part, les grimpeurs et les fédérations d'escalade en charge de les représenter.

Accès au Ramstein Intérieur du Ramstein dont laménagement a été soutenu par la commune de Baerenthal.

(Fig. 15)
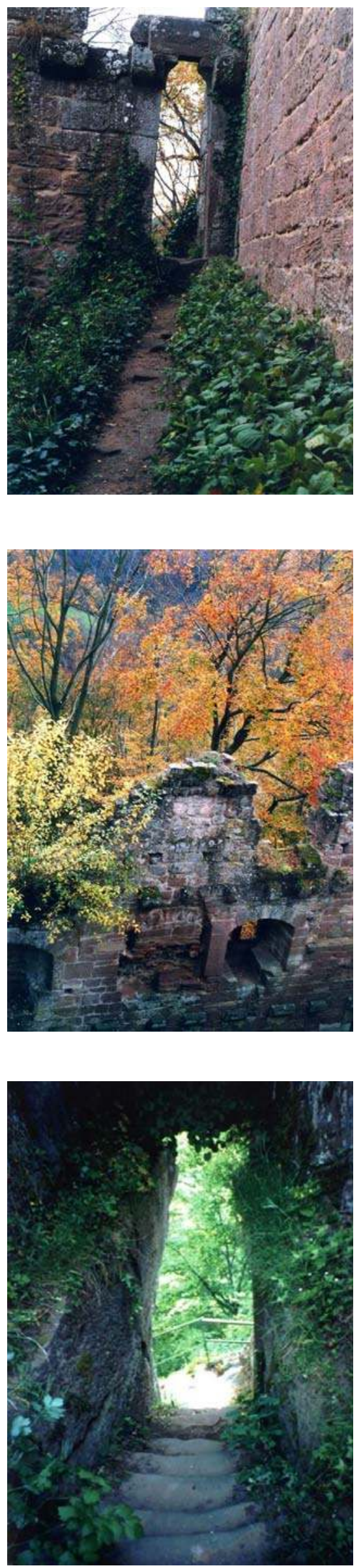


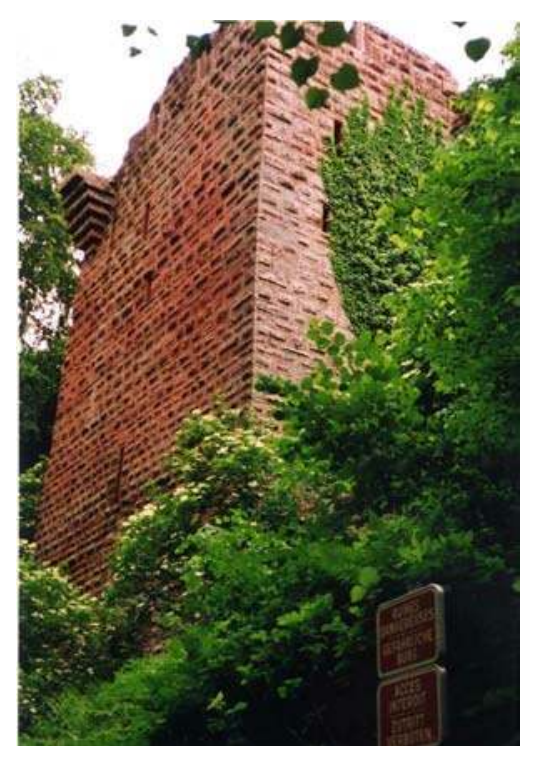

Le Falkenstein (Phillipsbourg)

La tour du puits du Falkenstein qui signifie « pierre du faucon ».

(Fig. 16)

\section{Le partage de la ruine: grimpeurs et faucons face à face}

En principe, tout utilisateur non professionnel de la forêt est tenu de se soumettre à des règles de respect de l'environnement: une certaine discrétion, des visites limitées à la période diurne, l'interdiction de faire des feux, de prélever des espèces animales et végétales, etc. À l'intérieur de ce cadre normatif, au titre cependant de la toujours problématique cohabitation des hommes et des bêtes, il est un cas de conflit d'intérêt qui prend un relief particulier puisqu'il a précisément pour théâtre les « nids d'aigle » que sont les ruines vosgiennes et les sommets rocheux: celui entre grimpeurs et faucons, ou plutôt protecteurs de faucons (Fig. 16 et 17).

Le faucon pèlerin affecte tout particulièrement les falaises gréseuses dans les cavités desquelles il niche au point d'être désigné comme l'oiseau emblématique des Vosges du Nord. D’une manière générale, du début des années 1970 jusqu'au milieu des années 1980, le faucon n'avait plus été observé dans les Vosges du Nord. À partir de cette date, on a constaté de nouveau sa présence. Une surveillance soutenue ayant probablement contribué à minorer les vols d'œufs, objets d'un trafic à destination des Émirats, où les pratiques cynégétiques réservent une grande place au faucon. Son « retour » n'a donc pas manqué de susciter l'intérêt des ornithologues, décidés à mettre en œuvre des mesures de protection de ce rapace inscrit sur la liste des espèces animales protégées au titre des articles L 211-1 et suivants du Code Rural, complétés par l'arrêté du 17 avril 1981, ainsi que par la Convention de Berne. Créée en 1994, l'association locale SOS Faucon pèlerin milite en faveur de sa repopulation et veille à garantir à l'oiseau des conditions optimales de vie et de reproduction. Le moment le plus sensible correspond à la nidification et au développement des oisillons, soit du début de l'année jusqu'à la fin du mois de juin, lorsque les jeunes faucons prennent leur envol et quittent définitivement le nid. SOS Faucon pèlerin s'est donc engagé dans une action de protection des zones notoirement investies par le faucon.

Or la seule présence humaine peut à terme faire fuir le faucon, comme cela s'est probablement produit au château du Falkenstein et au Fleckenstein, et de façon générale dans les sites à forte fréquentation. De fait, c'est moins le flux touristique, qui finalement n'occupe qu'un espace déjà partagé entre humains et animaux -, que la pratique d'escalade des rochers et de ruines a priori inaccessibles au tout public qui préoccupait les partisans et les responsables de la protection du rapace. En effet, dans un mouvement impulsé dans les années 1930 et qui s'est amplifié dans les années 1980, beaucoup de ruines et de rochers ont été équipés de pitons et des voies ont été ouvertes par des amoureux de la grimpe, de façon spontanée et sans concertation. Dans cette perspective, ruines et rochers constituent 
des espaces concurrentiels entre l'homme et l'animal. La cohabitation entre grimpeurs et faucons est d'autant plus problématique que l'un et l'autre sont politiquement - c'est-à-dire juridiquement - équipés : les porte-parole des faucons peuvent exciper de l'inscription sur la liste des espèces animales protégées - liste qui organise le droit envers et pour la nature -, les grimpeurs peuvent se prévaloir de la reconnaissance des activités physiques et sportives comme « droit pour tous » et « d'intérêt général », au titre de l'article 1 de la loi du 16 juillet 1984.

Soutenue par d'autres collectifs de protection de la nature et en accord avec les partenaires concernés, l'association SOS Faucon pèlerin a donc décidé de canaliser la pratique de ce sport en réglementant l'accès, dans le temps et dans l'espace, des principaux sites d'escalade où niche le faucon pèlerin, en particulier le Vieux-Windstein. Le responsable du groupement forestier des Vosges du Nord ${ }^{11}$ dans lequel se situe le Vieux-Windstein a longuement suivi l'affaire:

« Le Windstein, il a fait couler beaucoup d'encre et a attisé beaucoup d'animosité et de stress dans la région depuis quatre ans car le rocher représente $25 \%$ des capacités de grimpe dans les Vosges du Nord. C'est un site vraiment majeur sur lequel ils grimpaient sans aucune autorisation, aucune permission de qui que ce soit. Et puis, il y a quatre ans, le faucon pèlerin a décidé de nicher de nouveau dessus. C'est un animal qui niche très tôt, qui s'installe déjà mi-janvier - il n'y a pas de grimpeurs - le faucon a décidé de s'installer là-haut. C'est un oiseau protégé par la convention de Washington et tout le tralala. On a été tout de suite alerté par l'association comme quoi il fallait essayer de réglementer, de moduler, enfin d'interdire la grimpe sur le Windstein. Et puis, ils sont venus me voir. On s'est dit qu'on ferait tout pour le garder là-haut. Je leur ai dit que je suis tout à fait d'accord d'interdire, mais je trouve qu'il y a suffisamment de cailloux dans les Vosges du Nord pour qu'on se mette autour d'une table avec l'ensemble des propriétaires de rochers et qu'on dise: « on interdit pendant une certaine période mais on autorise d'autres rochers pour que les grimpeurs aient quelque chose ». J'avais à l'époque suggéré - et ça a été retenu - que ce soit le parc [Parc naturel régional des Vosges du Nord] qui gère la direction de cette réflexion. Ça a donné une charte, signée il y a deux ans. »

Après plusieurs mois de discussions et de concertations, un accord a donc été formalisé dans une Charte pour la pratique de l'escalade dans les Vosges du Nord, signée le 6 décembre 1997 par La Fédération française de la montagne et de l'escalade, l'association SOS Faucon pèlerin, l'ONF, les groupements forestiers concernés et le syndicat de coopération du Parc naturel régional des Vosges du Nord. La réglementation de l'accès aux sites se décline soit en interdiction pure et simple de la pratique de l'escalade - c'est le cas des rochers faisant partie des sites protégés par un arrêté préfectoral de protection de biotope ou classés en réserve naturelle volontaire, tel le Falkenberg, longue 
barre rocheuse située à proximité du Falkenstein -, soit en interdiction temporaire: ainsi, sur la ruine du Luzelhardt à Obersteinbach, les échelles d'accès sont retirées durant la période de nidification.

Sur la commune de Windstein, la ruine du Vieux-Windstein offre un cas intéressant de compromis entre la raison du loisir sportif et celle de la nature et de recomposition des intérêts des différentes parties représentées. En effet, cette ruine est un haut lieu d'escalade pour les Alsaciens et les Allemands qui la connaissent davantage sous le nom « Rocher de Windstein ». Mais des faucons y ont élu domicile depuis quelques années, sous l'œil attentif des ornithologues. Le site a donc été partagé en plusieurs secteurs dont certains sont formellement interdits durant la période sensible de nidification et redeviennent libres d'accès par la suite. Pour autant, la gestion du compromis reste toujours problématique. Le maire de Windstein estime que la mise en place de ces dispositions réglementaires a entraîné une baisse de la fréquentation du village; il remarque alors qu'elles privent le village d'une population de passage qui ne génère pas les effets négatifs du flux touristique: les grimpeurs sont des sportifs " extrêmement discrets », et en ce sens proches de la nature, ils représentent une forme de tourisme idéal, sorte d'écotourisme bienveillant pour l'environnement. Selon lui, une sensibilisation du public, sous la forme par exemple de journées à thème, servirait davantage la cause des faucons et permettrait que l'interdiction ne soit pas vécue comme arbitraire. Elle témoignerait du souci d'acquérir le soutien des acteurs locaux, et finalement contribuerait à accroître l'intérêt de la ruine du Vieux-Windstein.

\section{$\&$}

Que nous apprend cette scène locale du point de vue de la gestion des interfaces nature-culture? En guise de réponse et de conclusion, je voudrais faire deux brèves remarques. La première concerne la production de conflits qui éclatent autour de ces interfaces; la seconde invite à revenir sur l'une des modalités de gestion en émergence de ces objets de nature et culture: le site.

Les interfaces entre nature et culture sont conflictuelles. Elles le sont d'abord parce que les cultures occidentales pensent fortement la dichotomie nature-culture et instaurent une relation de concurrence entre ces deux mondes. Ce mode concurrentiel d'approche de ces objets sécants n'est pas obligatoirement lié au caractère remarquable des objets de nature et des pratiques qui les visent. Certaines pratiques agricoles sont dommageables pour des écosystèmes ou des ressources naturelles ordinaires, comme l'eau par exemple. Dans le cas des ruines, nature 
et culture non seulement se mêlent dans leurs pratiques, mais dans leur caractère à la fois ordinaire et remarquable. Si la forêt qui sert d'écrin à ces ruines émarge à une nature ordinaire pour cette région, la flore qui les peuple est suffisamment singulière pour mobiliser les botanistes contre les opérations de désherbage aveugle auxquelles ont parfois recours les restaurateurs de monuments historiques. Quant au faucon pèlerin, il est devenu un hôte de marque de ces pitons et pèse de tout son poids face aux grimpeurs qui déploient une autre pratique de la ruine. De la même manière, si les traces laissées par le passage des chars sur le sol gréseux de ces châteaux donnent accès à l'ancienne vie des lieux - la valeur d'ancienneté d'Aloïs Riegl - elles ne sont pas protégées au même titre que certains morceaux de bâtiments ou certains détails d'architecture - telle poivrière remarquable, telle fenêtre à ogive. C'est donc non seulement la combinaison de la nature et de la culture (de leurs répertoires pratiques propres et transposables) mais aussi l'enchevêtrement de leurs caractères ordinaires ou remarquables qui font la complexité de ces lieux. Cette imbrication singulière est également fortement productrice de conflits dès lors qu'une injonction patrimoniale occupe le cœur de la scène. Avec les ruines, l'injonction et la légitimité patrimoniale relèvent autant de préoccupations naturelles que culturelles; elles induisent par ailleurs un autre type d'exigence peut-être moins fréquemment identifié dans les politiques patrimoniales mais qui ressort nettement de l'enquête. Il s'agit de la revendication du libre accès au plus grand nombre de visiteurs. J'ai insisté sur ce point en parlant de ces ruines comme d'« objets démocratiques». L'expression, comme je l'ai indiqué, renvoie non seulement à la garantie d'accès au lieu pour le plus grand nombre de personnes mais aussi à la possibilité d'une grande diversité de pratiques de la ruine (de l'escalade aux jeux, en passant par la botanique ou la sortie historique).

Cette perspective de patrimonialisation élargie se retrouve dans la notion de « site » qui est aujourd'hui en émergence. Le site - dans son extension paysagère, touristique, archéologique, pittoresque qui peut être la ruine médiévale aussi bien que la friche et quel que soit le degré de clarté et d'officialité de son statut - est un espace, un support et un produit de patrimonialisation hybride. Il annonce une présence humaine passée ou à venir dont son organisation tient compte - ici, tenir compte du public et sécuriser la visite des ruines, voire également informer les visiteurs. Ouvert à tous contrairement à la réserve, il tient du « haut lieu » (Micoud 1991) sans faire forcément l'objet d'une approbation sociale consensuelle. Car un site rassemble autant qu'il divise les acteurs individuels ou collectifs qui le revendiquent; mais si ces acteurs en contestent parfois les qualités, la gestion ou les usages, c'est bien parce qu'ils s'y reconnaissent et y sont profondément attachés. Le site serait donc ainsi traversé par des forces a priori contraires mais qui trouvent à faire alliance, sinon alliage, c'est-à-dire non pas à se neutraliser mais à s'équilibrer dans la recherche d'ajustements des 
pratiques concernées. Il pourrait être, mais cela demande une exploration plus ample, l'un des modes de gestion émergent permettant d'administrer les biens de nature et de culture dans une unité de lieu et en tenant compte d'une diversité de pratiques et d'usages. Ce qui suppose, non seulement de réduire la disjonction nature culture mais aussi celle d'ordinaire et de remarquable.

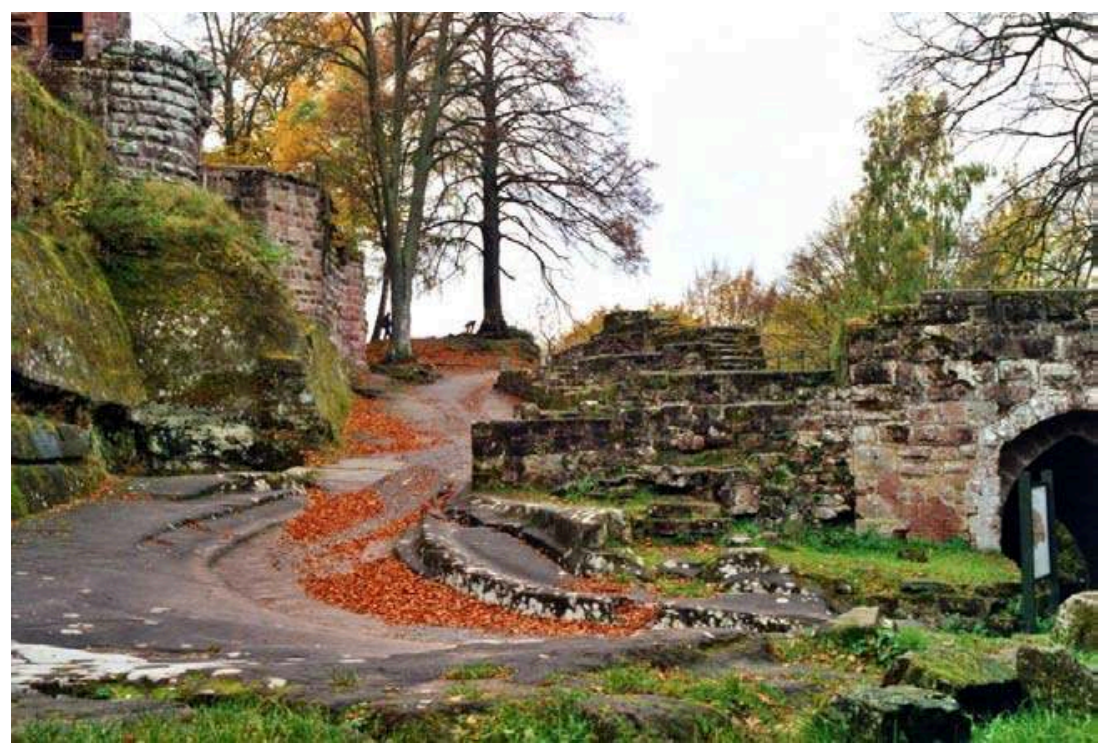

\section{Entrée du Fleckenstein}

Le Fleckenstein est un site touristique incontournable en matière de tourisme castral transfrontalier. Très bien restauré, il est aménagé pour recevoir le public qui sácquitte d'un droit d'entrée. L'alliance entre la nature et culture prend ici des formes différentes. Ses rambardes aux normes de sécurité, son entretien régulier en font un patrimoine culturel à ciel ouvert dont la pratique demande toutefois une certaine prudence.

(Fig. 18) 
Toutes les photos sont de l'auteur, sauf mention contraire.

Ces recherches ont été financées par la DRAC de Lorraine.

1. D'après la liste établie par le PNR (Parc naturel régional des Vosges du Nord 1980), on peut considérer que le corpus des châteaux forts des Vosges du Nord compte trente et un châteaux. Ces châteaux ont été édifiés pour la plupart entre le XIIe et le XIIIe siècle et détruits lors de la Guerre de Cent Ans. Certains appartiennent à la catégorie « château de forêt », c'est-à-dire qu'ils sont situés au cour d'un domaine forestier et accessibles seulement à pied, en empruntant les sentiers balisés du Club Vosgien. D'autres ressortissent à la catégorie " château de village »: proches des habitations, ils sont accessibles en quelques minutes.

2. Le PNR des Vosges du Nord s'étend sur deux régions, l'Alsace et la Lorraine. Situé à l'ouest de Strasbourg et à l'est de Bitche en Moselle, il est frontalier avec l'Allemagne.

3. La « valeur historique » d'un monument repose sur sa capacité à témoigner d'un stade de l'histoire (architectural, artistique, littéraire) et s'adresse essentiellement à un public d'experts équipés de références savantes. A contrario, la « valeur d'ancienneté » d'un monument est attachée « à la représentation du temps écoulé depuis sa création, qui se trahit à nos yeux par les marques de son âge. » (Riegl 1984 : 45). Le monument ancien, qui ne témoigne donc que d'« avoir subi l'épreuve du temps », est soumis à la loi de la dégradation naturelle, c'est-à-dire progressive et non imputable à une action humaine brutale.

4. C'est-à-dire dans le cas des ruines que j'ai appelées
« non productives» au sens marchand du terme.

5. G. Simmel est contemporain de A. Riegl, né comme lui en 1858. R. Debray présume qu'ils «ne se sont apparemment ni lus ni rencontrés » (Debray 1999 : 35). Pourtant dans un court texte intitulé « Réflexions suggérées par l'aspect des ruines », G. Simmel développe une analyse sur « la valeur esthétique de la ruine » très proche de celle d'A. Riegl.

6. Pour un inventaire floristique précis des châteaux vosgiens, on pourra se reporter à Walter 1938 et à Ulrich 2000 : 17-23.

7. Les ornières à l'entrée du Falkenstein sont une puissante évocation de la combinaison de ces deux éléments.

8. Parmi toutes les personnes que j'ai rencontrées, seule une jeune femme - ni résidente ni originaire des villages dont il est question - a clairement mentionné un rejet de la ruine: "C'est triste! elles sont toutes seules en pleine forêt; elles ont vécu et elles sont mortes. On dirait de grands animaux blessés ». Cette personne est, en revanche, passionnée d'églises et de cathédrales, qui ici s'opposent pleinement à la ruine.

9. Une Réserve biologique intégrale est l'un des outils juridiques permettant la protection des sites forestiers. Elle est destinée à laisser libre cours à la dynamique spontanée des habitats naturels, aux fins d'étude et de connaissance des processus impliqués, ainsi que de conservation ou développement de la biodiversité associée.

10. Sur les analogies entre la gestion du corps malade et celle des monuments, on se reportera à Foucart 1991.

11. Ce groupement forestier gère de nombreuses forêts privées concernées par des ruines. 


\section{RÉFÉRENCES}

Bischoff, Georges, 1989, « La paix des ruines », Saisons D’alsace, hiver: 53-70.

Davallon, Jean, 1991, " Produire des hauts lieux du patrimoine », in A. Micoud, (ed.), Des hauts lieux. La construction sociale de l'exemplarité. Paris, Éditions du CNRS: 85-102.

Dupré, Lucie, 2001, La Valeur patrimoniale des ruines. Expertise socioethnologique des ruines de châteaux médiévaux des Vosges du Nord. Rapport pour la direction régionale des Affaires culturelles de Lorraine.

Fabre, Daniel, 2000, « Ancienneté, altérité, autochtonie », in D. Fabre, (ed.), Domestiquer l'histoire. Ethnologie des monuments historiques. Paris, Éditions de la MSH: 195-208.

Foucart, Bruno, 1991, « Les sentiments de la ruine au XIX et au XXe siècle: tragicomédie en quatre actes », in Faut-il restaurer les ruines? Actes des colloques de la direction du Patrimoine. Paris, Direction du Patrimoine: 24-48.

Guillaume, Marc, 1980, La Politique du patrimoine. Paris, Galilée.

Huck, Joseph-Louis, 1972, « Le Club Vosgien de 1872 à 1918 », in Les Vosges et le Club Vosgien. Autour d'un centenaire 1872-1972. Strasbourg, Éditions le Club Vosgien: 233-255.

Jung, Edmond, 1990, « Contes et légendes d'Alsace », Les Vosges 1:2-6.

Micoud, André, 1991, « La production symbolique des lieux exemplaires », in A. Micoud, (ed.), Des hauts lieux. La construction sociale de l'exemplarité. Paris, Éditions du CNRS: 7-15.

Riegl, Aloïs, 1984 [1903], Le Culte moderne des monuments. Paris, Seuil.

Ruskin, John, 1989 [1880], The Seven Lamps of Architecture. New-York, Dover Publication.

Simmel, Georg, 1912, Mélange de philosophie relativiste, contribution à la culture philosophique. Paris, Félix Alcan.

Ulrich, Henri, 2000, La Légende des ruines. Arbres et châteaux des Vosges et du Jura alsacien. Strasbourg, La Nuée Bleue.

Walter, Émile, 1938, « La flore des châteaux féodaux en Vasgovie », extrait du Bulletin de la Société Nierderbronnaise d'Histoire et d'Archéologie 4. 


\section{RÉSUMÉ}

Conserver ensemble patrimoines naturel et culturel. Les ruines féodales des Vosges du Nord constituent un patrimoine exemplaire de l'intrication forte, complexe et parfois - mais pas systématiquement - problématique entre la nature et la culture. Hauts lieux de la culture locale mis en valeur dès la fin du XIXe par une association emblématique, elles portent en elles une tension particulièrement intéressante. En effet, d'un côté les ruines sont protégées en partie ou en totalité au titre des Monuments historiques et doivent par conséquent être l'objet de soins conservatoires attentifs. De l'autre, situées en pleine nature au cœur d'immenses territoires forestiers dont l'exploitation a constitué une des bases de l'économie locale, elles appartiennent à des gestionnaires sylvicoles qui n'ont ni les moyens ni les compétences pour s'employer à leur conservation.

Pour saisir la complexité de cet enchevêtrement entre intérêt naturel et intérêt culturel, il est intéressant de questionner ces ruines non seulement du point de vue des actions conservatoires qu'elles exigent mais également du point de vue de leur réception. En prenant appui sur la conceptualisation pionnière d'Aloïs Riegl, on intègre ainsi un acteur particulièrement décisif dans la question patrimoniale: le public. S'intéresser à la réception des ruines pose la question de leur partage et de leur appropriation par la diversité d'acteurs qui s'en revendiquent, qu'elles convoquent et/ou qui sont chargés de les entretenir et de les protéger.

Comment protéger dans le même temps la nature et la culture dans de tels sites précisément fondés sur l'imbrication de ces deux dimensions? Que faire lorsque les grimpeurs, qui ont leurs habitudes sur telle ruine devenue un site d'escalade réputé, se trouvent face au faucon pèlerin qui décide de revenir y nicher? Lanalyse de ce genre d'ajustements incite à revenir sur l'opposition tranchée entre nature et culture et conduit à considérer les arbitrages à chaque fois circonstanciés qui président au partage, à l'appropriation et à la conservation de ces sites.

\section{ABSTRACT}

Conserving Natural and Cultural Heritages Together. The feudal ruins in Northern Vosges constitute a heritage that epitomizes the strong, complex and sometimes - though not systematically- problematic entangling of nature and culture. As Meccas of local culture, valued since the end of the 19th century by an emblematic association, they manifest a particularly interesting tension. On the one hand, the ruins are partly or entirely protected as Historical Monuments and must consequently come under curators' attentive care. On the other hand, located in the wild, at the heart of huge forest areas whose exploitation constitutes one of the sources of the local economy, they belong to sylvicultural administrators who have neither the means nor the skills necessary for their conservation.

In order to grasp the complex overlapping between natural and cultural interests, these ruins must be examined both from the point of view of curators' actions that are necessary but also from the point of view of their reception. Based on the pioneer conceptualisation of Alois Riegel, a particularly decisive actor like the public is included in the heritage question. Interest in the reception of ruins raises the question of their appropriation and sharing by a variety of actors who claim, who are summoned and/or who are responsible for maintaining and protecting the ruins.

How can nature and culture be protected in such sites founded precisely on the intricate involvement of these two dimensions? What can be done when climbers with their own habits on ruins, well-known as climbing sites, find themselves faced with the peregrine falcon that has come to nest there? Analyzing this kind of adjustment calls for a revision of the clear-cut opposition between nature and culture and always leads to a consideration of detailed choices that govern the division, appropriation and conservation of these sites.

\section{MOTS CLÉS}

Vosges du Nord, Club Vosgien, monuments historiques, sylviculture, ruine médiévale, site, tourisme rural.

\section{KEYWORDS}

Northern Vosges, Club Vosgien, historical monument, sylviculture, medieval ruin, site, rural tourism. 